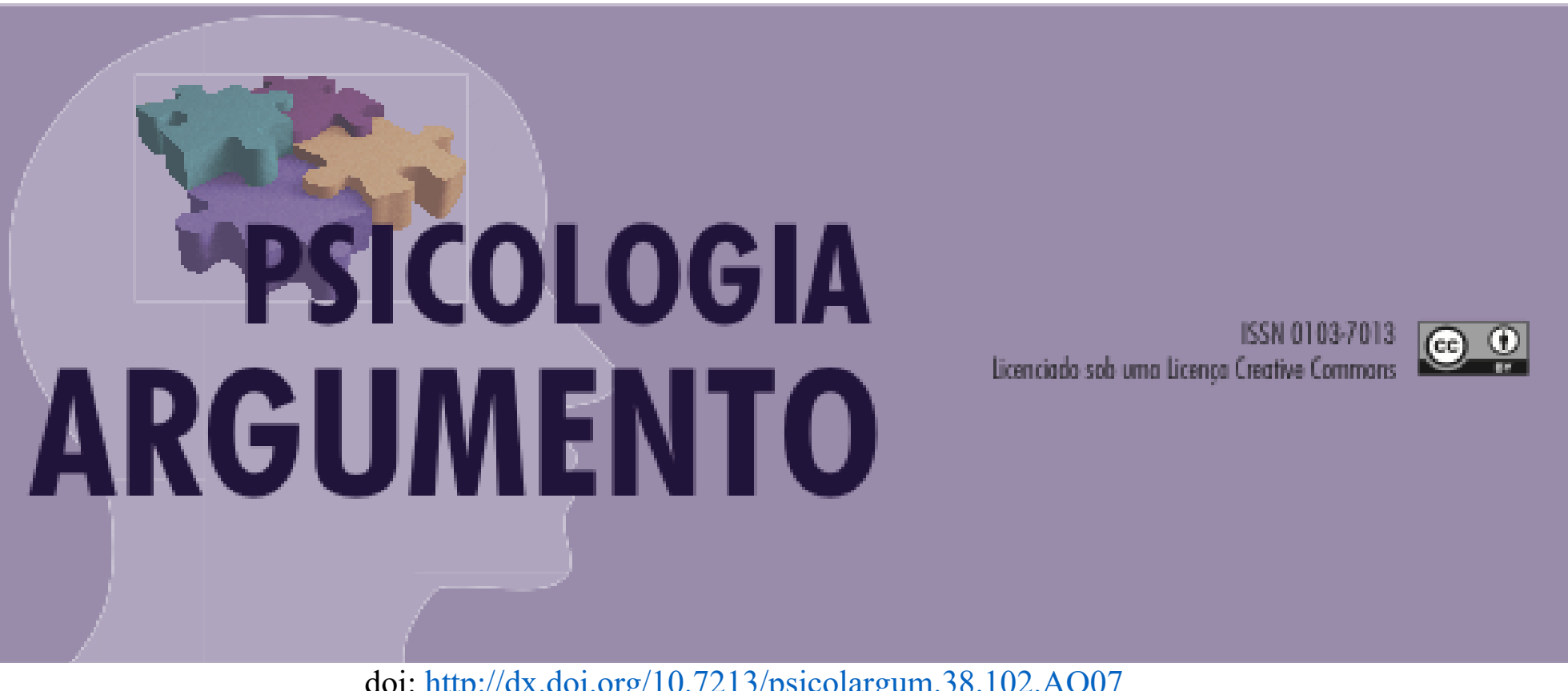

doi: $\underline{\text { http://dx.doi.org/10.7213/psicolargum.38.102.AO07 }}$

\title{
Representações Sociais do envelhecimento para mulheres que utilizam práticas de rejuvenescimento
}

Social representations of aging for women who use rejuvenation practices

Representaciones sociales del envejecimiento para mujeres que usan prácticas de rejuvenecimiento

\section{Amanda Castro}

Profa. Depto. Psicologia, Universidade do Extremo Sul Catarinense, Universidade Estácio de

Sá, e-mail: amandacastrops@gmail.com, orcid: https://orcid.org/0000-0002-8666-4494

Luiz Felipe Andrade Quadros

Residente do Programa de Residência Multiprofissional em Atenção Básica/Saúde da Família,

Universidade do Extremo Sul Catarinense, e-mail: luizfelipequadros@hotmail.com, orcid:

https://orcid.org/0000-0003-4448-7896

Marieli Mezari Vitali

Mestranda do Programa de Pós-Graduação em Psicologia, Universidade Federal de Santa

Catarina, e-mail: marielimezari@gmail.com, orcid: http://orcid.org/0000-0003-0052-7788

Camila Maffioleti Cavaler

Mestranda do P.P.G em Psicologia, Universidade Federal de Santa Catarina, e-mail: camilamaffioleticavaler@gmail.com,orcid: https://orcid.org/0000-0003-2417-8017

\section{Jacks Soratto}

Professor do Programa de Pós-Graduação em Saúde Coletiva, Universidade do Extremo Sul

Catarinense, e-mail: jacks@unesc.net, orcid: https://orcid.org/0000-0002-1339-7268

Brigido Vizeu Camargo

Professor do P.P.G. em Psicologia, Universidade Federal de Santa Catarina, e-mail: brigido.camargo@yahoo.com.br, orcid: https://orcid.org/0000-0002-9529-4923 


\title{
Resumo
}

O envelhecer se trata de um processo do ciclo da vida que caracteriza uma etapa marcada por mudanças físicas, psicológicas e sociais vivenciadas de forma particular em cada indivíduo. Nesse sentido, a Teoria das Representações Sociais se mostra essencial para acessar a perspectiva de diferentes grupos sobre esse fenômeno. O presente estudo buscou caracterizar as representações sociais do envelhecimento e as práticas de rejuvenescimento adotadas por mulheres. As participantes da pesquisa foram 40 mulheres, com idade entre 30 e 60 anos, que residiam na região da Grande Florianópolis. Do total de 40 mulheres, 20 utilizavam pelo menos um dos procedimentos rejuvenescedores não invasivos. As outras 20 mulheres faziam uso de pelo menos um dos procedimentos rejuvenescedores invasivos ou minimamente invasivos. A coleta de dados foi realizada através da técnica de associação de palavras com o termo indutor "envelhecimento", e um questionário contendo questões de caracterização das participantes e itens sobre as práticas de rejuvenescimento. Com relação ao conteúdo obtido sobre o envelhecimento a partir das evocações livres, ocorreram 200 evocações de 111 palavras diferentes. As palavras evocadas remetem às perdas e ganhos que envolvem o processo de envelhecimento. As representações sociais do envelhecimento apresentam aspectos comuns entre as participantes, e aspectos específicos, demonstrando que as práticas de rejuvenescimento adotadas possuem relação com a compreensão do envelhecimento para as mulheres. Tais dados permitem compreender que às representações sociais impactam na forma como se relacionam com suas aparências.

Palavras-chave: Representações Sociais; Envelhecimento; Rejuvenescimento.

\begin{abstract}
Aging is a process of the life cycle that characterizes a stage marked by physical, psychological and social changes experienced in a particular way in each individual. In this sense, the Theory of Social Representations is essential to access the perspective of different groups on this phenomenon. The present study sought to characterize the social representations of aging and the rejuvenation practices adopted by women. The research participants were 40 women, aged between 30 and 60 years old, who lived in the Greater Florianópolis region. Of the total of 40 women, 20 used at least one of the non-invasive rejuvenating procedures. The other 20 women used at least one of the invasive or minimally invasive rejuvenating procedures. Data collection was performed using the word association technique with the inducing term "aging", and a questionnaire containing questions on the characterization of participants and items on rejuvenation practices. Regarding the content obtained about aging from free evocations, there were 200 evocations of 111 different words. The evoked words refer to the losses and gains that involve the aging process. The social representations of aging present common aspects among the participants, and specific aspects, demonstrating that the rejuvenation practices adopted are related to the understanding of aging for women. Such data allow us to understand that social representations impact the way they relate to their appearances.
\end{abstract}

Keywords: Social Representations; Aging; Rejuvenation.

\begin{abstract}
Resumen
El envejecimiento es un proceso del ciclo de vida que caracteriza una etapa marcada por los cambios físicos, psicológicos y sociales experimentados de una manera particular en cada individuo. En este sentido, la Teoría de las representaciones sociales es esencial para acceder a la perspectiva de diferentes grupos sobre este fenómeno. El presente estudio buscó caracterizar las representaciones sociales del envejecimiento y las prácticas de rejuvenecimiento adoptadas por las mujeres. Los participantes de la investigación fueron 40 mujeres, con edades comprendidas entre 30 y 60 años, que vivían en la región del Gran Florianópolis. Del total de 40 mujeres, 20 utilizaron al menos uno de los procedimientos de rejuvenecimiento no invasivos. Las otras 20 mujeres usaron al menos uno de los procedimientos de rejuvenecimiento invasivos o minimamente invasivos. La recolección de datos se realizó utilizando la técnica de asociación de palabras con el término inductor "envejecimiento", y un cuestionario que contiene preguntas sobre la caracterización de los participantes y los items sobre prácticas de rejuvenecimiento. En
\end{abstract}


cuanto al contenido obtenido sobre el envejecimiento de las evocaciones libres, hubo 200 evocaciones de 111 palabras diferentes. Las palabras evocadas se refieren a las pérdidas y ganancias que implican el proceso de envejecimiento. Las representaciones sociales del envejecimiento presentan aspectos comunes entre los participantes y aspectos especificos, lo que demuestra que las prácticas de rejuvenecimiento adoptadas están relacionadas con la comprensión del envejecimiento para las mujeres. Dichos datos nos permiten entender que las representaciones sociales impactan la forma en que se relacionan con sus apariencias.

Palabras clave: Representaciones Sociales; Envejecimiento Rejuvenecimiento.

\section{Introdução}

O envelhecimento populacional vem alargando seus números ao longo dos anos. De acordo com a Organização Mundial da Saúde (OMS) e seus dados oferecidos em para a Organização Pan-Americana de Saúde (2018), entre 2015 e 2050 a proporção da população mundial com mais de 60 anos passará de $12 \%$ a $22 \%$, o que resulta em 2 bilhões, contraponto os 900 milhões em 2015. No Brasil, a estimativa é de que em 2030 tenha-se cerca de 113 milhões de mulheres (50,8\%) e 109 milhões de homens (49,2\%) na terceira idade (IBGE, 2016). Ou seja, uma população de idosos majoritariamente feminina.

Essa longevidade da população favorece oportunidades, tanto para a pessoa idosa, quanto para a sociedade. Esses indivíduos têm a possibilidade de buscar mais educação, uma nova carreira ou investimento na vida pessoal, de modo que a extensão dessas possibilidades são dependentes intrínsecas ao fator saúde. Considerando o conceito de saúde, como o bem-estar físico, mental e social, e não somente a ausência de enfermidade (OMS, 2006).

O envelhecer se trata de um processo do ciclo da vida que caracteriza uma etapa marcada por mudanças físicas, psicológicas e sociais vivenciadas de forma particular em cada indivíduo com sobrevida prolongada (Mendes, 2005). Nesse processo, surgem algumas perdas significativas, como a ocorrência de doenças crônicas deteriorantes, dificuldades financeiras, aposentadoria, viuvez, perdas de amigos, afastamento familiar, acontecimentos que impactam de maneira negativa na vida da pessoa idosa (Gatto, 2005). Na temática envelhecimento, inexiste consenso sobre quais são os aspectos determinantes de um bom envelhecer. Não obstante, há uma proeminente heterogeneidade sobre a percepção do processo de envelhecer pelos idosos (Teixeira \& Neri, 2008). 
Ao discutir essas características é necessário considerar as especificidades do envelhecer. Essa experiência se processa de modos diferentes para os gêneros, seja nos aspectos sociais, econômicos, condições de vida, doenças e até mesmo na subjetividade, de modo que essas diferenciações são fundamentais, principalmente porque determinam o lugar do idoso e da idosa na vida social. Os homens acabam apresentando menor baixo estima, enquanto as mulheres sentem maior autonomia, conquistam maior liberdade e buscam práticas de autocuidado (Figueiredo et al., 2007).

As representações sociais do envelhecimento são permeadas por vários aspectos, inclusive o de relações de gênero. A especificidade da situação de cada grupo social colabora para a especificidade das suas representações e essa especificidade concorre para a diferenciação dos grupos sociais (Moscovici, 1961/1976). Ainda que consideradas socialmente como vaidosas, com maiores práticas de autocuidado e relacionarem o envelhecimento à cuidados corporais estéticos, as mulheres tendem a perceber maior desfavorabilidade social em relação ao seu envelhecimento físico, quando se comparam aos homens (Castro, Antunes, Britto, \& Camargo, 2016).

Ao tratar da velhice, o setor de saúde opera com o maior número de práticas e intervenções, muitas vezes patologizando o processo de envelhecimento. Isso ocorre ao colocar no indivíduo que envelhece o desafio de manter a juventude e a jovialidade, através de uma estética que exige adequações para a permanência no mundo social, em especial para as mulheres, muitas, vítimas da ditadura do corpo e dos padrões de beleza (Cerqueira, 2017). Diante disso, esse grupo passa a aderir a procedimentos estéticos de rejuvenescimento. Goldenberg (2018) relata que as mulheres brasileiras, de forma semelhante às americanas, são as que mais utilizam procedimentos estéticos, como cirurgias plásticas, preenchimentos faciais, mudanças no cabelo, entre outros processos rejuvenescedores com a finalidade de melhorar a aparência no processo de envelhecimento.

A categorização dos procedimentos estéticos atualmente conhecidas são: procedimentos invasivos, minimamente invasivos e não invasivos (Murad, 2010). O Conselho Federal de Medicina (2016) descreve que procedimentos invasivos são aqueles que provocam o rompimento das barreiras naturais da pele ou penetram em cavidades do organismo, desse modo facilitam o acesso para o meio interno. A Sociedade Brasileira de Cirurgia Plástica (SBCP, 2009) caracteriza os procedimentos minimamente invasivos como aqueles que dispensam cirurgias e internalização, assim, não afetam a rotina do 
indivíduo. Também são caracterizados como procedimentos de mínima dor e baixo custo. Fazem parte desse grupo a dermoabrasão, laser, peeling químico, preenchimento cutâneo, toxina butolínica, plasma rico em plaquetas.

Cheung-Lucchese e Alves (2014) destacam que os recursos estéticos são indicados como uma solução fácil, prática e mais eficaz que as dietas restritivas e os exercícios físicos para a construção do corpo jovem. A medicalização da velhice sugere a compreensão do envelhecimento como um processo de declínio, que deve ser tratado. $\mathrm{Na}$ ciência do esporte, as perdas associadas ao envelhecimento também são tratadas, experimentos científicos apontam que determinadas intervenções por meio de exercícios físicos podem combater ou prevenir os declínios funcionais ligados ao envelhecimento. A ciência do esporte procura ser um aliado no combate ao envelhecimento, mas também abre a possibilidade para a reconstrução e ajuste do corpo em envelhecimento. No entanto, não há evidências suficientes que comprovem que o exercício possa remodelar corpos mais velhos. Nesse sentido, o exercício pode ser considerado como um recurso para propiciar um envelhecimento saudável com base na autonomia e bem-estar, vinculado ao estado subjetivo de sentir-se jovem (Tulle, 2008).

Identificar como as idosas compreendem o envelhecimento se faz necessário para entender esse processo. Nesse sentido, a Teoria das Representações Sociais se mostra essencial para acessar a perspectiva desse grupo, afinal, as representações sociais são uma forma de conhecimento elaborada e compartilhada socialmente com finalidade prática, que afeta a construção da realidade de um grupo (Jodelet, 2001).

As representações sociais são vinculadas a grupos, interligadas a seus aspectos sociais e culturais (Moscovici, 2012). Para Jodelet (2001), grupos compartilham uma visão consensual da realidade, que se diferencia ou assemelha às representações de outros grupos. Para a autora, as representações sociais além de interpretar e orientar condutas nas relações e experiências, também possuem a função de atuar no desenvolvimento de identidades pessoais e sociais, e influenciar a forma de se expressar e nas práticas sociais de indivíduos pertencentes a determinados grupos.

\section{Objetivos}

A partir da temática "Representações sociais do envelhecimento" esta pesquisa busca abranger as práticas de rejuvenescimento. O presente estudo buscou caracterizar as 
representações sociais do envelhecimento e as práticas de rejuvenescimento adotadas por mulheres.

\section{Método}

Esse estudo derivou de um macro projeto oriundo de uma dissertação, vinculado ao presente recorte uma abordagem qualitativa, de característica descritiva. As participantes da pesquisa foram 40 mulheres, com idade entre 30 e 60 anos, que residiam na região da Grande Florianópolis. Do total de 40 mulheres, 20 utilizavam pelo menos um dos procedimentos rejuvenescedores não invasivos, sendo estes: cosméticos, exercícios físicos aeróbicos, massagem modeladora e dieta alimentar. As outras 20 mulheres faziam uso de pelo menos um dos procedimentos rejuvenescedores invasivos ou minimamente invasivos tais como: injeção de toxina botulínica, cirurgias plásticas rejuvenescedoras, peelings químicos e bioplastia (preenchimento dérmico).

As práticas de rejuvenescimento foram selecionadas a partir de critérios fornecidos por uma pesquisa encomendada pela Sociedade Brasileira de Cirurgia Plástica acerca de procedimentos cirúrgicos e não cirúrgicos frequentemente utilizados no Brasil (SBCP, 2009). A categorização entre métodos invasivos, minimamente invasivos e não invasivos seguiu proposta de Murad (2012), em que os métodos invasivos ou minimamente invasivos são os que provocam o rompimento das barreiras naturais ou penetram em cavidades do organismo, já os procedimentos não invasivos são os que não envolvem instrumentos que rompem a pele ou que penetram fisicamente no corpo.

As participantes da pesquisa foram acessadas a partir de indicações de pessoas do convívio social dos pesquisadores, por meio da técnica metodológica bola de neve (snowball). Todas as participantes adotaram pelo menos uma das práticas de rejuvenescimento definidas para a pesquisa, no mínimo uma vez nos últimos seis meses, apresentando familiaridade com o objeto de estudo. A faixa etária foi estabelecida em função do período em que as marcas do envelhecimento tornam-se evidentes, a partir dos 30 anos (Papaléo Netto, 2002), sendo que se optou por estender a faixa etária da amostra até os 60 anos, pois é considerado o início da velhice no Brasil (Oliveira, 2019).

O grupo de mulheres que adota procedimentos não invasivos não fez em nenhum momento o uso de procedimentos invasivos (ou minimamente invasivos). No entanto, todas as mulheres que adotam práticas de rejuvenescimento invasivas ou minimamente 
invasivas já adotaram ou ainda adotam práticas não invasivas de rejuvenescimento. Não foram encontradas participantes que fizessem uso apenas de práticas invasivas ou minimamente invasivas de rejuvenescimento.

A coleta de dados foi realizada através da técnica de associação de palavras e um questionário contendo questões de caracterização das participantes e itens sobre as práticas de rejuvenescimento. As participantes do estudo foram convidadas a responder oralmente ao teste de evocação livre com a palavra "envelhecimento" como termo indutor. Foi solicitado que as participantes evocassem cinco palavras que lhes viessem "à cabeça" ao pensar em envelhecimento. Depois, foi solicitado que as participantes respondessem um questionário que compôs os dados de caracterização das participantes, incluindo: idade, situação conjugal, situação profissional e renda familiar. Além de questões fechadas acerca do comportamento passado das participantes em relação às práticas de rejuvenescimento, como os recursos utilizados e periodicidade de utilização de técnicas rejuvenescedoras.

Os dados obtidos por meio da técnica de associação de palavras foram submetidos a uma análise de conteúdo do tipo categorial, subdivida em três fases: pré-análise, exploração do material, inferência e interpretação (Bardin, 2011). Com o auxílio do software Atlas.ti (2020) para organização do corpus das evocações. A relação entre análise de conteúdo e o software Atlas.ti seguiu a proposta de Soratto, Friese e Pires (2020), com o agrupamento de conteúdos semelhantes para formação de categorias (elementos temáticos) e contagem de ocorrências.

As diretrizes legislativas vigentes que envolvem pesquisa com seres humanos foram respeitadas sendo aprovado pelo Comitê de Ética em Pesquisa da Universidade Federal de Santa Catarina sob o número 1.688.433. No momento da coleta foi ofertado o Termo de Consentimento Livre e Esclarecido (TCLE), assinado em duas vias, e garantido o anonimato das participantes.

\section{Resultados}

A média de idade das mulheres participantes da pesquisa foi de 42 anos (DP = 10,46 anos), sendo a idade mínima 30 anos e a idade máxima 60 anos. Em relação às práticas de rejuvenescimento adotadas nos últimos seis meses, destacam-se 26 pessoas 
que utilizaram cosméticos e 14 que praticaram exercícios físicos aeróbicos, conforme destacado na Tabela 1.

Tabela 1

Distribuição das práticas de rejuvenescimento adotadas

\begin{tabular}{cccc}
\hline Prática de rejuvenescimento & $\begin{array}{c}\text { Mulheres que } \\
\text { adotam práticas } \\
\text { invasivas } \begin{array}{c}\text { Mulheres que } \\
\text { minimamente } \\
\text { invasivas }\end{array}\end{array}$ & $\begin{array}{c}\text { adotam apenas } \\
\text { práticas } \\
\text { Não invasivas }\end{array}$ & Total \\
\hline Cosméticos & 18 & 8 & 26 \\
Exercícios físicos & 6 & 8 & 14 \\
Cirurgias plásticas & 10 & 0 & 10 \\
Dieta alimentar & 2 & 5 & 7 \\
Massagens modeladoras & 2 & 3 & 5 \\
Preenchimento dérmico & 5 & 0 & 5 \\
Injeção de toxina botulínica & 2 & 0 & 2 \\
Peeling químico & 2 & 0 & 2 \\
\hline
\end{tabular}

Todas as pessoas que adotaram práticas invasivas ou minimamente invasivas, concomitantemente fazem uso de outras práticas de rejuvenescimento não invasivas $(n=20)$, mas para fins de comparação foram incluídas apenas no grupo que adota práticas invasivas e minimamente invasivas. A variável "tipo de prática de rejuvenescimento" foi agrupada em duas modalidades, associada à faixa-etária, conforme a Tabela 2.

Tabela 2

Distribuição das participantes segundo práticas de rejuvenescimento adotadas de acordo com a faixa etária

\begin{tabular}{cccc}
\hline & \multicolumn{2}{c}{$\begin{array}{c}\text { Quantidade de mulheres que adotam } \\
\text { práticas de rejuvenescimento }\end{array}$} & \\
\cline { 2 - 4 } Faixa etária & $\begin{array}{c}\text { Práticas invasivas e } \\
\text { minimamente } \\
\text { invasivas }\end{array}$ & $\begin{array}{c}\text { Apenas práticas } \\
\text { Não invasivas }\end{array}$ & Total \\
\hline $30-39$ & 8 & 11 & 19 \\
$40-49$ & 3 & 5 & 8 \\
$50-60$ & 9 & 4 & 13 \\
\hline Total & 20 & 20 & 40 \\
\hline
\end{tabular}

No que se refere à escolaridade das participantes, a maior parte $(\mathrm{n}=21)$ frequentou até o ensino superior completo, sendo que 19 participantes concluíram o ensino médio. A renda familiar mensal média foi de 7,27 salários mínimos $(\mathrm{DP}=1,78)$, havendo predominância dessa média entre o grupo de mulheres que adotam práticas invasivas ( $\mathrm{n}$ 
$=14$ ). Foi verificado ainda que 5 pessoas eram aposentadas, 2 donas de casa e as outras 33 desenvolviam atividades laborais remuneradas.

Em relação ao estado civil, 23 pessoas eram casadas, 14 eram solteiras e 3 divorciadas. Sobre a existência de filhos, 25 mulheres afirmaram possuir filho(s). Foi investigado também com quem a participante convive em sua casa e os resultados indicam a predominância de uma configuração familiar formada por companheiro e filho(s) $(\mathrm{n}=14)$.

Em relação às fontes de informação sobre as práticas de rejuvenescimento, a maioria $(\mathrm{n}=17)$ utiliza a internet como principal fonte. Além disso, também foram citados os folhetos de produtos $(n=6)$, familiares e amigos $(n=6)$, televisão $(n=5)$, revistas $(n=4)$ e a opinião de especialistas $(n=2)$.

Com relação ao conteúdo obtido sobre o envelhecimento a partir das evocações livres, ocorreram 200 evocações de 111 palavras diferentes. Posteriormente, as palavras evocadas pelas participantes foram categorizadas, considerando a revisão de literatura, no intuito de agrupar aquelas que representassem um mesmo elemento semântico das Representações Sociais (RS) sobre o envelhecimento. Para melhor visualização as frequências foram registradas de acordo com o tipo de prática de rejuvenescimento adotada. A partir das palavras evocadas foram criadas 10 categorias, conforme ilustra a Tabela 3.

Tabela 3

Categorias elaboradas a partir das evocações obtidas com o termo indutor Envelhecimento

Tipo de prática de rejuvenescimento adotada

Categoria

$\begin{array}{ccc}\text { Práticas invasivas e minimamente } & \text { Práticas } & \text { Total } \\ \text { invasivas } & \text { Não invasivas } & \\ \boldsymbol{f} & \boldsymbol{f} & \boldsymbol{f}\end{array}$

\begin{tabular}{lccc}
\hline Transformações fisiológicas & 18 & 29 & 47 \\
Ganhos subjetivos & 12 & 21 & 33 \\
Desassistência & 18 & 6 & 24 \\
Transformações estéticas & 19 & 3 & 22 \\
Perdas subjetivas & 9 & 10 & 19 \\
Suporte social & 6 & 11 & 17 \\
Caracterizações etárias individuais e grupais & 6 & 9 & 15 \\
Finitude e espiritualidade & 2 & 8 & 10 \\
Disponibilidade de tempo e dinheiro & 6 & 3 & 9 \\
Atividades físicas & 4 & 0 & 200 \\
\hline \multicolumn{1}{c}{ Total } & 100 & 100 & 2 \\
\hline
\end{tabular}


As categorias serão descritas a seguir, considerando a ordem hierárquica estabelecida a partir da frequência de evocações. Para o termo indutor "Envelhecimento", a categoria mais frequente foi "Transformações fisiológicas", com 47 evocações. Nessa categoria estão presentes elementos que remetem ao desgaste orgânico decorrente do envelhecimento, são citadas doenças e limitações funcionais. Como pertencentes a essa categoria é possível destacar os elementos: doença $(f=15)$; perda de vitalidade $(f=4)$; dor $(f=4)$; menopausa $(f=3)$; perda de funções $(f=2)$. Tal categoria apresentou maior frequência de evocações entre as participantes que adotam práticas de rejuvenescimento não invasivas $(f=29)$.

A categoria "Ganhos subjetivos" $(f=33)$ foi composta majoritariamente por palavras evocadas por mulheres que adotam práticas de rejuvenescimento não invasivas. Essa categoria abrange características pessoais e de personalidade que aparecem como ganhos advindos do processo de envelhecimento. São representantes desta categoria as palavras experiência $(f=10)$, maturidade $(f=4)$ e sabedoria $(f=3)$.

Como pertencentes à categoria "Desassistência" $(f=24)$ é possível destacar as palavras solidão $(f=10)$, preconceito $(f=3)$ e rejeição $(f=2)$. Tais palavras remetem a situações de desassistência e desamparo social, sendo as evocações principalmente presentes entre o grupo de mulheres que adota práticas de rejuvenescimento invasivas e minimamente invasivas.

A categoria "Transformações estéticas" $(f=19)$ possui palavras evocadas principalmente por mulheres que adotam práticas de rejuvenescimento invasivas e minimamente invasivas. Abrangendo palavras como rugas $(f=5)$, cabelos brancos $(f=3)$ e flacidez $(f=2)$, essa categoria engloba mudanças corporais relativas à aparência ao longo do processo de envelhecimento.

A categoria "Perdas subjetivas" $(f=19)$ é formada por sentimentos e características psicológicas negativas que são atribuídas ao envelhecimento. São palavras que constituem essa categoria: depressão $(f=5)$, desânimo $(f=2)$ e medo $(f=2)$. Em contrapartida, a categoria "Suporte social" $(f=17)$ abarca palavras que correspondem à rede social de apoio daquele que está em processo de envelhecimento, como filhos $(f=3)$, família $(f=2)$ e netos $(f=1)$.

$\mathrm{Na}$ categoria "Caracterizações etárias individuais e grupais" $(f=15)$ estão presentes palavras como idoso $(f=3)$, velho $(f=2)$ e terceira idade $(f=2)$. Nessa categoria 
são destacados termos etários popularmente utilizados para denominação do indivíduo que envelhece e do grupo ao qual este pertence.

Palavras como morte $(f=2)$, Deus $(f=1)$ e finitude $(f=1)$ estão presentes na categoria "Finitude e espiritualidade" $(f=10)$. Essa categoria é formada por evocações realizadas principalmente por mulheres que adotam práticas não invasivas de rejuvenescimento. Trata da terminalidade prevista ao fim ou durante o envelhecimento e enfatiza conteúdos religiosos e espirituais relativos ao pós-morte.

"Disponibilidade de tempo e dinheiro" $(f=9)$ é uma categoria que trata da aposentadoria e planos futuros que envolvem o tempo e condições financeiras na velhice. Palavras como aposentadoria $(f=2)$, condições financeiras $(f=1)$ e viagem $(f=1)$, ilustram o conteúdo presente nessa categoria.

Por último destaca-se a categoria "Atividades físicas" $(f=4)$, que abrange atividades relacionadas à manutenção da saúde orgânica a partir da movimentação do corpo, sendo citada unicamente pelo grupo de mulheres que adotam práticas de rejuvenescimento invasivas e minimamente invasivas. Compõem essa categoria palavras como exercícios $(f=1)$, atividades físicas $(f=1)$ e ginástica $(f=1)$.

\section{Discussão}

A categoria mais frequente nas evocações foi "transformações fisiológicas" relacionadas com o envelhecimento. Demonstrando que, essas mulheres, principalmente as adeptas às práticas de rejuvenescimento não invasivas, associam em suas representações o envelhecer com as mudanças de seus corpos de uma maneira negativa, com enfoque nas perdas físicas. Em consonância com estes resultados, dados obtidos por Fernandes de Araújo, Coutinho e Saldanha (2005) apontam que os idosos, principalmente aqueles portadores de doenças crônicas, objetivam suas representações sociais da velhice com uma conotação negativa e comumente associada ao binômio: velhice-doença. Esse aspecto negativo, também surgiu na pesquisa de Carvalho (2018), em que em um grupo de 70 mulheres com idade entre 40 e 75 anos, as pertencentes ao grupo da meia-idade atribuíram um número maior de doenças ao envelhecimento, sugerindo uma percepção negativa do mesmo.

Percebe-se, com isso, uma preocupação com o envelhecer de forma saudável. $\mathrm{Na}$ pesquisa de Faria et al. (2019) que investigou a qualidade de vida de idosos, no grupo 
formado por quatro mulheres de 60 a 80 anos, surgiu essa atenção com o envelhecimento de forma ativa e saudável, de modo que isso possa lhes proporcionar bem-estar psicossocial e, também, maior longevidade. Esses dados também podem ser observados em uma amostra mais jovem, na pesquisa de Carrara, Vinagre e Pereira (2020) que visou analisar a percepção de mulheres que buscam por procedimentos estéticos sobre o envelhecimento, com uma amostra de 51 mulheres de meia-idade (40 a 59 anos) e 19 idosas (60 anos ou mais), mostrou que as mulheres de meia-idade têm uma percepção mais negativa sobre o envelhecimento do que as mulheres idosas, atribuindo um número mais elevado de alterações na saúde ao envelhecimento, sugerindo uma crença negativa a esse processo.

Em contrapartida, em nossa pesquisa, o mesmo grupo de mulheres, que adotam práticas de rejuvenescimento não invasivas, também descreveram um relevante número de evocações sobre o envelhecimento como um "ganho subjetivo". Revelando assim uma face externa do envelhecer ligada a mudança fisiológica e uma relação com a doença; e outra face, positiva, relacionada ao que o passar dos anos agrega à experiência de desenvolvimento pessoal, como o saber e a maturidade.

Dado semelhante foi observado na pesquisa de Pecora, Anjos e Paredes (2010), em que apesar das dificuldades, temores e angústias que surgem no processo de envelhecimento, foi percebida a presença de satisfação no envelhecer, e sua carga de aprendizado subjetivo obtido com o passar dos anos. No estudo de Teixeira et al. (2007), é salientada a relação entre perdas e ganhos, no grupo de mulheres acima de 60 anos é apresentada uma avaliação positiva do objeto a partir de elementos como sabedoria, maturidade, experiência, paz e amor.

No estudo de Fernandes e Garcia (2010), que objetivou a análise do sentido da velhice, um grupo com 18 mulheres expressou a velhice de modo ambíguo, apesar de entenderem a velhice como algo negativo, para muitas mulheres essa fase representa a oportunidade de desfrutar seus próximos anos de vida de forma mais livre. Fin et al. (2017) em sua pesquisa com uma amostra de 10 mulheres de 60 a 69 anos, identificaram a associação da velhice a uma etapa positiva da vida, com estima à autonomia física e mental, independência e integração à beleza da experiência vivida.

É relevante essa análise da percepção positiva e negativa do envelhecimento, principalmente no que diz respeito aos efeitos psicológicos. Em um estudo que envolveu 288 participantes com mais de 66 anos, o sofrimento psicológico e a percepção positiva 
do envelhecimento apresentou um impacto positivo direto na qualidade de vida dos sujeitos (Ingrand et al, 2018).

Essa categoria de representação é muito semelhante a de "perdas subjetivas", essa, com um número de evocação similar nos dois grupos de mulheres, na qual o medo e sintomas psicológicos de desânimo e estado de depressão, surgem como efeito subjetivo do envelhecer. Resultado semelhante foi encontrado na pesquisa de Teixeira et al. (2007), no qual um grupo de mulheres entre 25 e 35 anos apresentou predominância dos aspectos negativos sobre o envelhecimento, a partir de elementos como medo, depressão e perda de autoestima.

Esse sofrimento, é passível de observação, nas evocações das mulheres adeptas a práticas de rejuvenescimento invasivas e minimamente invasivas, que atribuiriam evocações que representam "desassistência" no envelhecimento. O receio do abandono, preconceito e solidão marca o discurso dessas mulheres. Evidenciando um sofrimento, não apenas de cunho causal a questão fisiológica, mas também psicossocial.

Evocações sobre a família, que compõem a categoria de "suporte social", também marcam a importância dos vínculos no envelhecer, devido o anseio de manter as relações e amenizar esse medo da desassistência. Corroborando com a discussão de Fernandes e Andrade (2016) que o apoio familiar é um elemento que ajuda o idoso no processo de enfrentamento dos conflitos emergentes na velhice.

No estudo de Brito et al. (2018) a velhice foi associada à saída dos filhos de casa, e acaba se relacionando com o estado de solidão, e a necessidade de voltar para perto dos mesmos. Brito, Camargo e Castro (2017) analisaram em uma amostra de 80 pessoas, entre elas idosos e seus familiares, e também constataram a presença de elementos de desvalorização da velhice, expressa no desrespeito ao idoso e na própria avaliação da velhice que utiliza os parâmetros da juventude.

Esses temores relacionados ao envelhecimento podem justificar a busca por processos de rejuvenescimento, pois, no grupo de mulheres adeptas às práticas de rejuvenescimento invasivas e minimamente invasivas, emergiram evocações associadas à "transformações estéticas" diretamente associadas à aparência no envelhecer. Essa questão estética do envelhecer tem sido prezada na atual sociedade, de modo que o envelhecer com beleza está relacionado com juventude e saúde. Os procedimentos estéticos surgem como alternativa para essas mudanças e destaca-se como grande público as mulheres (Witczak, Zamberlan, \& Ssparemberger, 2013). 
As mudanças físicas relacionadas diretamente à estética têm uma consequência na identidade pessoal da mulher idosa, como o não reconhecimento do seu corpo. De modo que a centralidade da compreensão de beleza, no sentido de perda, está associada a dicotomia que envolve o tempo e a qualidade da imagem do seu corpo (Veloz, Nascimento-Schulze, \& Camargo, 1999). Camargo, Justo e Jodelet (2010) discutem o porquê dessa preocupação estética feminina e abordam uma distinção das representações e práticas sociais entre homens e mulheres, em que se verifica maior submissão às normas sociais por parte das mulheres. Elas acabam sendo mais valorizadas em virtude da sua atratividade física relativa à jovialidade, enquanto aos homens são consideradas outras características para ascensão social, como situação financeira e profissional.

A questão estética apresentada nessa pesquisa também foi evidenciada por Mello (2019) em uma amostra de 11 mulheres entre 60 a 80 anos. A fim de investigar a percepção da mulher idosa sobre a estética na velhice, identificou que a aparência para esse grupo é percebida como um importante aspecto de bem-estar próprio e em relação a outros, assim como gerador de confiança e felicidade, mas não ocupa um lugar de destaque no cotidiano.

A busca do bem-estar é perceptível nas poucas evocações que compõe a categoria "atividade física", composta exclusivamente por falas das mulheres adeptas a procedimentos de rejuvenescimento invasivos ou minimamente invasivos. O que demonstra que à atenção ao corpo pelo viés de promoção à saúde através de práticas físicas, é pouco associado ao envelhecimento. Em contrapartida, Miranda e Santiago (2020) ao investigarem o processo de envelhecimento e sua relação com a atividade física, considerando que esta se apresenta como uma estratégia na busca de um sujeito ativo e saudável, concluíram que mesmo com limitações físicas, é possível que o idoso se mantenha ativo e autônomo.

No mesmo viés, Biasus, Demandova e Camargo (2011), em uma pesquisa com 40 homens e 40 mulheres investigaram as representações sociais do envelhecimento e da sexualidade para pessoas com mais de 50 anos. A representação do envelhecimento aparece associada a autonomia no exercício de atividades, de modo que para esse público, se torna relevante a participação em grupos de atividade e lazer para manter-se ativos, tendo uma maior aderência à essas práticas o grupo feminino.

Outra questão emergente, apesar de poucas evocações, e em maior número entre as mulheres que adotam práticas de rejuvenescimento invasivas e minimamente 
invasivas, é a preocupação com planos futuros, questões financeiras e aposentadoria. Nesse mesmo sentido, a pesquisa de Wachelke et al. (2008) sobre representações sociais do envelhecimento, com uma amostra de 496 indivíduos com idade entre 15 e 59 anos identificou que quanto mais avançada a idade dos participantes, mais surgem representações de aposentadoria e novas ocupações.

Assim como a aposentadoria é uma temática que surge com maior frequência com o avançar da idade, a questão da finitude da vida e espiritualidade emergem como temáticas que permeiam as representações das participantes. Corroborando com esse dado, a pesquisa de Fernandes e Andrade (2016) com um grupo de idosos que representam a velhice como uma etapa que se direciona para a temporalidade e finitude do ser humano, fazendo levantar a questão do sentido da vida, assim como a urgência em viver. O estudo apontou que alguns idosos buscam na fé elementos de consolo, de proteção e também de esperança.

Percebe-se que as mulheres que fazem uso de práticas de rejuvenescimento não invasivas foram as que mais ofereceram evocações relacionadas aos processos fisiológicos e ganhos subjetivos em relação ao envelhecimento. Cabe considerar que esse grupo teve em sua composição a predominância de mulheres mais jovens, de 30 a 39 anos, o que pode representar um certo otimismo em relação ao envelhecimento.

Em contrapartida, não houve neste grupo nenhuma evocação na categoria de atividade física, informação intrigante, diante da importância da prática dessas atividades nos processos fisiológicos do envelhecer. Fator que se mostra importante no estudo de Santana e Maia (2009), em que a atividade física além de ser reportada com a sensação de bem-estar, também repercute no aspecto físico-motor e na saúde de forma geral dos idosos.

Identifica-se que o grupo de mulheres que adotam práticas de rejuvenescimento invasivas e minimamente invasivas, com o número maior de mulheres entre 50 a 60 anos, tem suas representações sociais do envelhecimento mais associadas a transformações estéticas, o que justifica a busca por tratamentos mais invasivos. Bem como a questão da desassistência, que de acordo com as pesquisas abordadas nesse artigo (Teixeira et al., 2007; Fernandes \& Andrade, 2016; Brito et al, 2018), é uma preocupação relevante com o passar da idade, atrelada aos efeitos psicológicos e perdas que esse anseio resulta. Apesar desse receio do desamparo, essas mulheres foram as que menos fizeram 
evocações relacionadas a finitude e espiritualidade, e a preocupação com os laços sociais pode se converter na percepção da preocupação com o presente nessa etapa de suas vidas.

De modo geral, podemos perceber que as palavras evocadas diante do termo indutor "envelhecimento" remetem às perdas e ganhos que envolvem o processo de envelhecimento. Essas perdas e ganhos abrangem maior disponibilidade de tempo com a chegada da aposentadoria, desassistência e desamparo social, bem como a rede de suporte social e as transformações estéticas e fisiológicas ocorrentes no corpo até a chegada da velhice, com consequente percepção de finitude.

\section{Considerações finais}

O objetivo da pesquisa foi compreender as representações sociais do envelhecimento por mulheres que adotam práticas de rejuvenescimento. Identificou-se que as mulheres que adotam práticas de rejuvenescimento não invasivas associam o envelhecimento principalmente as mudanças fisiológicas, de modo específico, ao processo de funções típicas do processo de envelhecimento, como o surgimento de doenças e limitações físicas. Sendo possível identificar que as representações sociais do envelhecimento estão fortemente relacionadas com características negativas desse processo. No entanto, também foi possível identificar entre o mesmo grupo de mulheres a associação do envelhecimento com ganhos subjetivos decorrentes do mesmo, como experiência, maturidade e sabedoria, evidenciando outro lado, este positivo, do envelhecer para essas mulheres.

Mulheres que adotam práticas de rejuvenescimento invasivas e minimamente invasivas associam o envelhecimento a mudanças estéticas como rugas, cabelos brancos e flacidez, ao contrário do outro grupo de mulheres. O que justificaria a busca de práticas de rejuvenescimento invasivas, considerando a preocupação estética mais acentuada. Esse mesmo grupo de mulheres evocaram mais palavras relacionadas a desassistência, evidenciando a preocupação com o desamparo social que o envelhecimento pode trazer, o que também justificaria a adoção de práticas estéticas para minimizar os efeitos do envelhecer.

As representações sociais do envelhecimento apresentam aspectos comuns entre as participantes, e aspectos específicos, de acordo com o tipo de prática adotada pelas participantes: não invasivas ou invasivas e minimamente invasivas. Mostrando que as 
práticas adotadas de rejuvenescimento possuem relação com a compreensão do envelhecimento para as mulheres deste estudo, ou seja, essas representações sociais impactam na forma como se relacionam com suas aparências.

\section{Referências}

Bardin, L. (2011). Análise de conteúdo. Lisboa: Edições 70 (Trabalho original publicado em 1977).

Biasus, F., Demantova, A., \& Camargo, B. V. (2011). Representações sociais do envelhecimento e da sexualidade para pessoas com mais de 50 anos. Temas psicol. 19(1) 319-336, Recuperado de: http://pepsic.bvsalud.org/scielo.php?script=sci_arttext\&pid=S1413$389 \times 2011000100025 \& \operatorname{lng}=$ pt\&nrm=iso

Brito, A. M. M., Camargo, B.V., \& Castro, A. (2017) Representações Sociais de Velhice e Boa Velhice entre Idosos e Sua Rede Social. Revista de Psicologia da IMED, 9(1) 5-21. doi:https://doi.org/10.18256/2175-5027.2017.v9i1.1416.

Brito, A. M. M. et al. (2018). Representações sociais do cuidado e da velhice no Brasil e Itália. Psic.: Teor. e Pesq., 34, e3455. https://doi.org/10.1590/0102.3772e3455.

Camargo, B. V., Justo, A. M., \& Jodelet, D. (2010). Normas, representações sociais e práticas corporais. Interamerican Journal of Psychology, 44(3), 449-457. Recuperado de: https://www.redalyc.org/articulo.oa? $\mathrm{id}=28420658006$

Carrara, F. F., Vinagre, C. G. C. M., \& Pereira, L. P. (2020) Percepção do envelhecimento: mulheres de meia idade e idosas que buscam por procedimentos. Id on Line Rev. Mult. Psic. 14(49) 38-50. https://doi.org/10.14295/idonline.v14i49.2309

Carvalho, F. F. C. R. (2018) Análise da percepção do envelhecimento em mulheres de meia idade e idosas que buscam por procedimentos estéticos. Dissertação de Mestrado. Universidade de Santo Amaro - UNISA. São Paulo.

Cerqueira, M. B. (2017). Míticas do envelhecimento: em busca de uma vida saudável. Ciências Sociais Unisinos. 53(1):148-157. doi: 10.4013/csu.2017.53.1.15

Conselho Federal de Medicina (2016). PARECER CFM 35/2016 Procedimentos invasivos na dermatologia. Retrieved 10 Jun 2020, from https://sistemas.cfm.org.br/normas/arquivos/pareceres/BR/2016/35_2016.pdf

Faria, L. C., Santos, K. H., Sousa, M. S., Anunciação, C, Silva, A. P., \& Oliveira, J. F. (2019). Envelhecimento, Relações de Gênero e Qualidade de Vida da População Idosa. Anais do $16^{\circ}$ Congresso Brasileiro de Assistentes. Brasília. Recuperado de: http://broseguini.bonino.com.br/ojs/index.php/CBAS/article/download/167/165/ 
Fernandes de Araújo, L., Coutinho, M. P., \& Saldanha, A. A. W. (2005). Análise comparativa das representações sociais da velhice entre idosos de instituições geriátricas e grupos de convivência. Psico, 36(2), 197-204. Recuperado de: http://revistaseletronicas.pucrs.br/ojs/index.php/revistapsico/article/view/1390/1090

Fernandes, J. S. G., \& Andrade, M. S. (2016). Representações sociais de idosos sobre velhice. Arq. bras. psicol. 68(2), 48-59. Recuperado de: http://pepsic.bvsalud.org/scielo.php?script=sci_arttext\&pid=S180952672016000200005

Fernandes, M. G. M., \& Garcia, L. G. (2010). O Sentido da Velhice para Homens e Mulheres Idosos. Saúde Soc. 19(4), 771-783, 2010. https://doi.org/10.1590/S010412902010000400005.

Figueiredo, M. L. F. et al. (2007) As diferenças de gênero na velhice. Rev. bras. enferm., 60(4) 422-427. https://doi.org/10.1590/S0034-71672007000400012.

Fin, T. C., Portella, M. R., \& Scortegagna, S. A. (2017). Velhice e beleza corporal das idosas: conversa entre mulheres. Revista Brasileira de Geriatria e Gerontologia, 20(1), 74-84. https://doi.org/10.1590/1981-22562017020.150096

Gatto, I. B. Aspectos Psicológicos do Desenvolvimento. In: Netto, Matheus Papaléo. Gerontologia: a velhice e o envelhecimento em visão globalizada. (2005). São Paulo, Atheneu, 2005. p.109-113.

Goldenberg, M. (2018). Mirian. The invention of a beautiful old age: in search of a freer, happier life. Rev. bras. geriatr. gerontol.. 21(5), 511-512, doi: https://doi.org/10.1590/1981-22562018021.180177.

Ingrand, I. et al. (2018). Positive perception of aging is a key predictor of quality-of-life in aging people. PLoS One, 13(10), e0204044. doi: https://doi.org/10.1371/journal.pone.0204044 O

Instituto Brasileiro de Geografia e Estatística. (2016). IBGE Projeções. Recuperado de: www.ibge.gov.br/apps/populacao/projecao/

Jodelet, D. (2001). Representações sociais: um domínio em expansão. In: D. Jodelet. (Org.). As representações sociais. Rio de Janeiro: Ed Uerj.

Mello, M. de. (2019). Estética na velhice: a percepção da mulher idosa. Dissertação (Mestrado em Envelhecimento Humano) - Universidade de Passo Fundo, Passo Fundo, Rio Grande do Sul.

Mendes, M. R.S.S. B., Gusmão, J. L., Faro, A. C. M., \& Leite, R. C. B O. (2005). A situação social do idoso no Brasil: uma breve consideração. Acta Paulista de Enfermagem, 18(4), 422-426. https://doi.org/10.1590/S0103-21002005000400011. 
Miranda, F. B., \& Santiago, L. M. (2020). O cuidado de si e o envelhecimento: uma análise em 2017 a partir de um programa de saúde pública na cidade do Rio de Janeiro. J Manag Prim Health Care. https://doi.org/10.14295/jmphc.v12i0.562

Moscovici, S. (2012). A psicanálise, sua imagem e seu público. Petrópolis, RJ: Vozes. (Obra original publicada em 1961).

Murad, A. (2012). Dermatologia Cosmética. Rio de Janeiro: Elsevier editores.

Oliveira, A. S. (2019). Transição Demográfica, Transição Epidemiológica e Envelhecimento Populacional no Brasil. 2019. Revista Brasileira de Geografia Médica e da Saúde, 15(31): 69-79. doi: http://dx.doi.org/10.14393/Hygeia153248614

Organização Mundial da Saúde. (2006). Constituição da Organização Mundial da Saúde. Documentos básicos. Recuperado de https://www.who.int/governance/eb/who_constitution_sp.pdf.

Organização Pan-Americas de Saúde (OPAS). (2018). Folha Informativa Envelhecimento. Recuperado de: https://www.paho.org/bra/index.php?option=com_content\&view=article\&id=5661:f olha-informativa-envelhecimento-e-saude $\&$ Itemid $=820$.

Papaléo Netto, M. (2002). Gerontologia: a velhice e o envelhecimento em visão globalizada. São Paulo: Atheneu.

Parente, E. L.M. (2016, 14 Sep 2016). O que são procedimentos minimamente invasivos? [Weblog]. Retrieved 9 July 2020, from https://sbcp-sc.org.br/artigos/o-que-saoprocedimentos-minimamente invasivos/\#: :text=Em\%20linhas\%20gerais $\% 2 \mathrm{C} \% 20$ procedimentos $\% 20$ minimament e,chances $\% 20 \mathrm{de} \% 20$ resultar $\% 20 \mathrm{em} \% 20$ complica $\% \mathrm{C} 3 \% \mathrm{~A} 7 \% \mathrm{C} 3 \% \mathrm{~B} 5 \mathrm{es}$.

Pecora, A. R., dos Anjos, P. M., \& Paredes, E. C. (2010). O envelhecimento como processo social. Revista de Educação Pública, 19(39), 55-73. Recuperado de: http://cacphp.unioeste.br/extensao/unati/arqs/ UNATI_11.pdf

Santana, M. da S. \& Maia, E. M. C. (2009). Atividade Física e Bem-Estar na Velhice. Revista de Salud Publicar Recuperado de https://scielosp.org/article/rsap/2009.v11n2/225-236/

Sociedade Brasileira de Cirurgia Plástica (2009). Cirurgia Plástica no Brasil. Pesquisa Datafolha, Janeiro. Recuperado de: http://www2.cirurgiaplastica.org.br/wpcontent/uploads/2012/11/pesquisa2009.pdf.

Soratto, J., Pires, D. E. P., \& Friese, S. (2020). Thematic content analysis using ATLAS.ti software: Potentialities for researchs in health. Revista Brasileira de Enfermagem, 73(3), 1-5. https://doi.org/10.1590/0034-7167-2019-0250 
Teixeira, I. N. D. O., \& Neri, A. L. (2008). Envelhecimento bem-sucedido: uma meta no curso da vida. Psicol. USP., 19 (1) 81-94. https://doi.org/10.1590/S010365642008000100010 .

Teixeira, M. C. T. V., Settembre, F. M., \& Leal, S. B. (2007). A survey of women's social representations of aging and rejuvenation. The Spanish Journal of Psychology, 10(1), 104-114. DOI: $10.1017 / \mathrm{s} 1138741600006363$

Veloz, M. C. T., Nascimento-Schulze, C. M., \& Camargo, B. V. (1999). Representações sociais do envelhecimento. Psicol. Reflex. Crit., 12(2), 479-501. https://doi.org/10.1590/S0102-79721999000200015.

Wachelke, J. F. R., Camargo, B. V., Hazan, J. V., Soares, D. R., Oliveira,L. T. P., \& Reynaud, P. D. (2008). Princípios organizadores da representação social do envelhecimento: dados coletados via internet. Estudos de Psicologia (Natal). http://dx.doi.org/10.1590/S1413-294X2008000200002.

Witczak, P. E., Zamberlan, L., \& Sparemberger, A. (2013). O envelhecer e a beleza feminina: significados e comportamentos de consumidoras de produtos associados. In: XXXIII Encontro Nacional de Engenharia de Produção. A Gestão dos Processos de Produção e as Parcerias Globais para o Desenvolvimento Sustentável dos Sistemas Produtivos. Salvador, BA, Brasil, 08 a 11 de outubro de 2013.

Submetido em: 08/05/2020

Aprovado em: 22/07/2020 